\title{
Study on kinetics of thermal degradation of Polymethyl Methacrylate/Cyclic Olefin Copolymer blend
}

\author{
Maryam Khosravinezhad*, Masoumeh Kazemi \\ Department of Polymer Engineering, Faculty of Chemical Engineering, Shiraz branch, Islamic Azad University, Shiraz, Iran \\ * Corresponding author, e-mail: mkhosravi243@yahoo.com
}

Maryam Khosravinezhad: https://orcid.org/0000-0001-6135-3473

Masoumeh Kazemi: https://orcid.org/0000-0001-9756-4096

\begin{abstract}
In this investigation, polymethyl methacrylate (PMMA) was mixed with cyclic olefin copolymer (COC) because of its hardness, strength, and transparency properties. The results of thermal analysis through TGA and DTG showed that the thermal properties of the alloy are improved using $40 \%$ cyclic olefin copolymer. Kinetics of thermal degradation (pyrolysis) of polymers have been studied and analyzed and thermal pyrolysis of polymethyl methacrylate and cyclic olefin copolymer thermoplastic polymer was conducted. The computation of kinetic analysis is measured along with the different methods used to study the kinetics. The activation energy $(E)$ of degradation of studied materials was estimated using Ozawa Flynn and Wall (OFW), Starink and Kissinger's methods, and evaluation of three kinetic parameters taken appropriate kinetic model in terms of percent change for both types of polymers have been proposed, and finally, simulated curves were compared with the experimental curves. Both mechanisms of degradation for COC and PMMA under nitrogen atmosphere will reflect intramolecular transfer and random scission of the main chain.
\end{abstract}

Keywords: Pyrolysis, Kinetics, Thermal degradation, PMMA, COC.

\section{Introduction}

Synthetic polymers have gained a great deal of attention since 1940, and various industries have been transformed by petroleum-based polymers such as polyethylene, polypropylene, polystyrene, polyethylene terephthalate, polymethyl methacrylate, polyvinyl chloride, etc. But in the late nineteenth century, humans were able to make plastics by transforming polymers. The variety of plastics allows them to be used in a variety of applications, from simple components such as plastic bags and bottles to the manufacture of car parts, computer parts, electronic equipment, and more [1].

Global production of plastics is more than 368 million tons per year, and almost half of it is discarded after a short period of use and remains in nature for more than 30 years [2]. Plastics based on petrochemical products such as polyolefins, polyesters, and polyamides due to their availability in large quantities, low price and desirable performance characteristics, such as good tensile strength and tear, resistance to oxygen and organic compounds, and thermal sealing capabilities, increasingly used as packaging plastics [3].

Plastics based on acrylic and methacrylate monomers are known as acrylic plastics. The most important acrylic plastics are poly (methyl methacrylate) which is produced by polymerization of methyl methacrylate. Polymethyl methacrylate is one of the well-known polymers that with $92 \%$ light transmittance and suitable color, which is unaffected by time and climate change, is a serious competitor to glass in many applications [4]. $\mathrm{COC}$ is an engineered thermoplastic copolymer with a combination of unique properties. The COC form in the process has a basic carbon chain that has no double bonds [5]. Alloying existing polymers and copolymers is one of the most interesting and cost-effective methods and is usually much more economical and achieves the desired result faster than other methods. Polymethyl methacrylate with cycloolefin copolymer (COC), due to having some similar properties, forms a two-component alloy. Mechanical properties show that with increasing cycloolefin copolymer to polymethyl methacrylate at low percentages of cycloolefin copolymer, properties such as impact resistance and elongation and tensile and flexural strength are reduced due to incompatibility and insufficient miscibility. To stabilize the morphology of the alloy or greater compatibility between the two components of the alloy, new fields of polymer irradiation, irradiation of polymer alloys are used [6]. Alloying existing polymers and copolymers is one of the most interesting and cost-effective methods and is usually much more economical and achieves the desired result faster than other methods [7].

Over the last few decades, research has focused on modifying existing polymers to create new materials with desirable properties. Due to achieving a wide range of properties during the alloying of two or more polymers, this method is known as one of the best applied methods to improve the properties of polymers. An alloy of two or more compatible polymers is a method of producing materials with a set of unique properties. To obtain the best two-polymer alloy, there must be an appropriate degree of mutual compatibility. There are several methods 
to study the compatibility of polymers in their alloys. Methods include differential dynamic calorimetry (DSC) [8], thermal-mechanical analysis (DMA) [9], dielectric analysis [10], mechanical properties such as tensile strength [11], optical methods such as refractive index determination [12], thermal expansion measurement [13], morphological studies using TEM [14], SEM [15], etc.

The thermal stability of polymers depends on a variety of factors. Because, thermal resistance is a function of bond energy, when the temperature reaches a point that causes the bonds to rupture, the polymer breaks down through vibrational energy. Use structures that contain the strongest chemical bonds, such as, heteroaromatic compounds, aromatic ethers, and the use of weakly bonded structures such as alkylene-alicyclic and unsaturated hydrocarbons are recommended. The structure of the compound must be such that it tends to be stable, its resonant stability is high, and finally, the circular structures must have a normal bond length, so that if a bond is broken, it holds the main structure of the atoms together [16].

Polymer degradation is a destructive process that must be avoided. The process that can be effective in preventing or delaying this degradation is called polymer stabilization. To create such a high-performance resistor, we need to understand the mechanism of polymer degradation, identify the factors that cause it, as well as the factors that cause stability in polymers. Sometimes, but not always, the destruction of polymers can be beneficial. De-polymerization results in the formation of many monomers that can be used for the practical fabrication of these materials [16].

To calculate the kinetic parameters, either at different temperatures or different speeds, the conditions under consideration is set. Kinetics examines the measurement and parametric parameters at different reaction rates. The three parameters of activation energy (E), conversion percentage (a), and thermal degradation order reaction (n) are called triple kinetic parameters [17]. In addition to these values, other influential parameters such as heat rate $(\beta)$, temperature $(\mathrm{T})$, and conversion percentage function $\mathrm{f}(\mathrm{a})$ are also considered in kinetic calculations. In kinetic calculations, the conversion rate $(\mathrm{a})$ is defined as $\mathrm{a}=\left(\mathrm{w}_{0}-\mathrm{w}_{\mathrm{t}}\right) /\left(\mathrm{w}_{0}-\mathrm{w}_{\mathrm{f}}\right)$ and $\mathrm{w}_{0}, \mathrm{w}_{\mathrm{t}}, \mathrm{w}_{\mathrm{f}}$ is the initial, real, and final weights of the sample in this equation, respectively. In this research, the heat degradation kinetics of pure polymethyl methacrylate sample, pure cyclic olefin copolymer sample and PMMA/COC40 alloy before and after irradiation at different heating rates have been investigated.

\section{Materials and Methods}

In this study, polymethyl methacrylate with grade CM-205 and melt flow index of $1.8 \mathrm{~min} \mathrm{~g} / 10$ was prepared from CHI MEI Taiwan, and Cyclic olefin copolymer was purchased from the German company TOPAS, with grade 6015, some of the specifications of which are given in Table 1.

\subsection{Preparation of the samples}

In the first step, PMMA and COC were mixed manually to prepare the sample, and samples were prepared by combining 5, 10, 20, and 40 weight percent percentages of COC. PMMA and COC were dried in a heated oven for 6 hours at $80{ }^{\circ} \mathrm{C}$ and physically mixed. Alloying was done using an isotropic two-screw extruder. The process temperature was $260-230^{\circ} \mathrm{C}$ and its speed was set around $200 \mathrm{rpm}$. A vacuum pump was also placed in the extruder to remove residual air during alloying. Then, extruded, granulated and dumbbell samples were prepared for tensile, bending, and impact testing according to ASTM standard, with the KRAUSS MAFFEI KM injection molding machine. For all samples, injection conditions were set as an injection temperature of 260-230 ${ }^{\circ} \mathrm{C}$, mold temperature of $70{ }^{\circ} \mathrm{C}$ and an injection pressure of $30 \mathrm{MP}_{\mathrm{a}}$. Finally, the samples were dried in a vacuum oven at $70{ }^{\circ} \mathrm{C}$ for 2 hours, and then stored in a dry place at an ambient temperature of about $27 \pm 2{ }^{\circ} \mathrm{C}$. Based on the results of physical-mechanical tests, the optimal alloy sample, PMMA / COC40 was determined. In this study, thermal analysis was performed on the optimal sample before and after irradiation [6].

\subsection{Thermometry (TGA)}

The sample is hung inside a platinum or alumina plant in the furnace. The end of the hanging wire is connected to a magnetic core that enters or leaves the coil cylinder due to weight change. This electric current core was induced in proportion to the change in sample weight in the coil and after amplification was sent to the recording part. The sample temperature was also measured with a thermocouple near and under the plant where the sample is located. In the recording part, the induced current in the coil, which is proportional to the change in sample weight, the vertical axis, and the electric current generated in the thermocouple as the horizontal axis, plotted the weight change curve for temperature. The weight of the sample required in the TGA method was 20 to $200 \mathrm{mg}$. The furnace environment was static; gas was flowing, or maybe airless. It was thin with a length of 15-20 and a diameter of 3-5 cm. The weight measuring part in today's devices has a sensitivity of about one microgram and therefore the device should generally be safe from environmental vibrations. The result of the thermal weighing test was a weight change curve in terms of temperature. 


\subsection{Derivative Thermogravimetry (DTG)}

In a thermal weighing test, if the temperatures of two thermal events are close to each other, it is difficult to separate them in the weight change curve in terms of temperature. The problem is that by adding an electronic part to the TGA device, the derivative of the weight curve can be plotted in terms of temperature. This is called the Derivative Thermogravimetry (DTG). The DTG curve provides TG information visually more easily. It also allows the maximum weight loss temperature (TMax) to be determined quickly, resulting in $\mathrm{T}$ (onset) and $\mathrm{T}$ (endset) information. All three temperatures change with changing test conditions, and the area below the DTG curve is directly proportional to the change in mass.

\subsection{Methods for evaluating kinetic parameters}

To calculate the kinetic parameters, $30 \mathrm{mg}$ of each sample (pure sample of polymethyl methacrylate, pure sample of cyclic olefin copolymer and sample of PMMA / COC40 alloy, before and after irradiation) was used in TGA thermal analysis test. Thermal analysis was performed under temperature conditions of $25-700{ }^{\circ} \mathrm{C}$ at heating rates of 5,10 , and 20 in nitrogen medium.

\subsection{Calculation of activation energy}

Flynn-Wall-Ozawa, Starink and Kissinger methods were used to calculate the activation energy. In fact, by this analysis, the dependence or non-dependence of the activation energy (E) on the conversion percentage of (a) was determined. If (E) was independent of (a), the thermal degradation process was one-step and simple. But if the activation energy changed with the conversion percentage, the pyrolysis process was complex and so-called multi-step. Therefore, the shape of the $(\mathrm{E}-\mathrm{a})$ curve predicted the reaction mechanism. In these methods, temperatures proportional to the percentage of constant conversion at different heating rates $(\beta)$ were measured and finally, the diagram $\ln (\beta)$ vs. $(1 / \mathrm{T})$ was drawn. Activation energy $(\mathrm{E})$ was calculated by determining the slope of this linear diagram. If the activation energy changed with the conversion percentage, multi-step reaction mechanisms prevailed [18].

Flynn-Wall-Ozawa (Eq. 1):

$$
\ln \left(\beta_{i}\right)=\text { Const }-1.052\left(\frac{E_{a}}{R T_{a}}\right)
$$

Kissinger (Eq. 2):

$$
\ln \left(\frac{\beta_{i}}{T_{a, i}^{2}}\right)=\text { Const }-\left(\frac{E_{a}}{R T_{a}}\right)
$$

Starink (Eq. 3):

$$
\ln \left(\frac{\beta_{i}}{T_{a, i}^{1.96}}\right)=\text { Const }-1.0008\left(\frac{E_{a}}{R T_{a}}\right)
$$

\subsection{Calculation of kinetic parameters of PMMA}

To calculate the kinetic parameters of polymethyl methacrylate, kinetic model-dependent methods, nth-order reaction method and Málek method were used [19].

\subsection{1. nth-order reaction method}

In the nth-order reaction method, a kinetic model $f(\alpha)=(1-\alpha)^{n}$ is used [20] (Eq. 4).

$$
\frac{d \alpha}{d t}=A \exp \left(\frac{-E_{a}}{R T}\right)(1-\alpha)^{n}
$$

When $\beta$ heating rate and $\mathrm{dT}=\beta \mathrm{dt}($ Eq. 5,6$)$ :

$$
\begin{gathered}
\frac{d \alpha}{d T}=\frac{A}{\beta} \exp \left(\frac{-E_{a}}{R T}\right)(1-\alpha)^{n} \\
F(\alpha)=\int_{0}^{\alpha} \frac{d \alpha}{(1-\alpha)^{n}}=\frac{A}{\beta} \int_{T_{0}}^{T} \exp \left(\frac{-E_{a}}{R T}\right) d T=\frac{A}{\beta} G(T)
\end{gathered}
$$

To calculate the $\mathrm{G}(\mathrm{T})$ function of the temperature integral, the MATLAB program was used, and finally, the kinetic Eq. $(7,8)$ for the analysis of the simulated DTG diagram was obtained as follows: 


$$
\begin{gathered}
\frac{d \alpha}{d t}=\beta \frac{d \alpha}{d T} \\
\frac{d \alpha}{d T}=\frac{A}{\beta} e^{-c / T}\left\{1+(1-n) \frac{A}{\beta}\left[\left(T \exp \left(\frac{-c}{T}\right)-c E_{1}\left(\frac{c}{T}\right)\right)-\left(T_{0} \exp \left(\frac{-c}{T_{0}}\right)-c E_{1}\left(\frac{c}{T_{0}}\right)\right)\right]\right\}^{\left(\frac{n}{1-n}\right)}
\end{gathered}
$$

And by placing $c=E_{a} / R$, the simulated TGA diagram is obtained from the following Eq. (9):

$$
\begin{aligned}
1-\alpha=\left\{1+(1-n) \frac{A}{\beta}\left[\left(T \exp \left(\frac{-E_{a}}{R T}\right)-\left(\frac{E_{a}}{R}\right) E_{1}\left(\frac{E_{a}}{R T}\right)\right)\right.\right. \\
\left.\left.\left.-\left(T_{0} \exp \left(\frac{-E_{a}}{R T_{0}}\right)-\left(\frac{E_{a}}{R}\right) E_{1}\left(\frac{E_{a}}{R T_{0}}\right)\right)\right]\right\}\right\}^{\left(\frac{1}{1-n}\right)}
\end{aligned}
$$

After solving this equation, kinetic parameters $(E, \ln A, n)$ were obtained to simulate the TG and DTG diagrams.

\subsubsection{Málek method}

In the Málek method to obtain the appropriate kinetic model, the model was selected based on the function $\mathrm{y}(\alpha)$ and using Table 2. According to the Málek model, the activation energy (E) was obtained through conversion methods or fitting methods.

Parameter (n) was calculated from the following Eq. (10):

$$
\alpha_{p}=1-\left(1+\frac{1-n}{n} x_{p} p\left(x_{p}\right)\right)^{1 / n-1}
$$

When $\alpha_{p}$ was the conversion percentage at the $T_{p}$ point (peak temperature of the DTG diagram), $x_{p}=E_{a} / R_{p}$ and $\mathrm{p}\left(\mathrm{x}_{\mathrm{p}}\right)$ were obtained using the Senum and Yang approximation [21] (Eq. 11).

$$
p(x)=\frac{e^{-x}}{x}\left(\frac{x^{3}+18 x^{2}+88 x+96}{x^{4}+20 x^{3}+120 x^{2}+240 x+120}\right)
$$

Therefore, parameter (A) was calculated as follows (Eq. 12):

$$
A=-\frac{\beta x_{p}}{T_{p} f^{\prime}\left(\alpha_{p}\right)} \exp \left(x_{p}\right)
$$

The term $f^{\prime}\left(\alpha_{p}\right)$ is the first-order derivative of $f(\alpha)$ at the point $\alpha_{p}: f^{\prime}(\alpha)=-n(1-\alpha)^{n-1}$.

After calculating the kinetic parameters, to solve the following differential Eq. (13), the ode45 function was used in the program using the Rung-Kutta method [22].

$$
\frac{d \alpha}{d T}=\frac{A}{\beta} \exp \left(\frac{-E_{a}}{R T}\right)(1-\alpha)^{n}
$$

\subsection{Calculation of kinetic parameters of COC}

To calculate the COC kinetic parameters, kinetic model dependent methods, Málek method and nonlinear fitting method were used.

\subsubsection{Málek method}

The following suitable kinetic model was used (Eq. 14, 15, 16):

$$
\frac{d \alpha}{d T}=\frac{A}{\beta} \exp \left(\frac{-E_{a}}{R T}\right) \alpha^{m}(1-\alpha)^{n}
$$

$$
\alpha_{m}=\alpha(\text { Mpo int })
$$




$$
\begin{aligned}
P & =\frac{\alpha_{m}}{1-\alpha_{m}} \\
\ln \left[\left(\frac{d \alpha}{d t}\right) e^{x}\right] & =\ln A+n \ln \left[\alpha^{p}(1-\alpha)\right]
\end{aligned}
$$

Drawing a graph $\ln \left[\left(\frac{d \alpha}{d t}\right) e^{x}\right]$ versus $\ln \left[\alpha^{p}(1-\alpha)\right]$ and $\alpha €(0.2$ and 0.8$)$, a straight line was obtained, through which the slope of the parameter $n$ was obtained.

And the parameter $m$ was obtained as follows:

$\mathrm{m}=\mathrm{pn}$

After calculating the kinetic parameters, the ode45 function was used to solve the differential equation $\mathrm{m}$ in the MATLAB program using the Rung-Kutta method.

\subsubsection{Nonlinear fitting method}

In this method, to obtain the kinetic parameters, the following Eq. (17) was used:

$$
\chi^{2}=\sum_{j=1}^{N}\left[\frac{(d \alpha / d T)^{\exp }-(d \alpha / d T)^{\text {calc }}}{\sigma_{j}}\right]^{2}
$$

Using Levenberg-Marquardt nonlinear fitting algorithm [23], the above equation was solved and the kinetic parameters were obtained. The activation energy was also calculated using the Kissinger method.

\subsection{Evaluation of kinetic parameters of PMMA/COC40 alloy}

To calculate the kinetic parameters of PMMA/COC40 alloy before and after irradiation, two methods, nth-order reaction (for PMMA) and Málek (for COC) were used together.

$$
\begin{gathered}
\frac{d \alpha}{d t}=\Sigma \mathcal{W}_{1}\left(\frac{d \alpha_{1}}{d t}\right)+w_{2}\left(\frac{d \alpha_{2}}{d t}\right) \\
\frac{d \alpha}{d t}=A \exp \left(\frac{-E_{a}}{R T}\right)(1-\alpha)^{n}+A \exp \left(\frac{-E_{a}}{R T}\right) a^{m}(1-\alpha)^{n}
\end{gathered}
$$

In solving the part of the kinetic equation by the Málek method for the second peak, related to COC in the alloy, the activation energy was obtained through the Kissinger method.

\section{Results}

3.1. Calculation of activation energy for pure PMMA

3.1.1. Kinetic parameters by nth-order reaction method

To calculate the kinetic parameters by the nth-order reaction method, after solving the equations, the kinetic parameters $(E),(\ln A),(n)$ were obtained to simulate the TG and DTG diagrams. Fig. 1 shows the TG diagram obtained from the laboratory data and the diagram simulated by the nth-order reaction method at thermal rates of 5,10 , and $20{ }^{\circ} \mathrm{C} / \mathrm{min}$.

Considering the PMMA peaks (Fig. 1,2) at the thermal rates in Table 3, it was concluded that with increasing the thermal rate, the maximum degradation temperature also increased. At high thermal rates, degradation occurred at relatively high temperatures.

\subsubsection{Kinetic parameters by Málek method}

In the Málek method, the y $(\alpha)$ function was used to obtain the appropriate kinetic model. The kinetic parameters determined for pure PMMA were obtained using Málek and nth-order reaction methods based on the data obtained from Fig. 3 and 4.

\subsection{Calculation of activation energy for pure COC}

\subsubsection{Kinetic parameters by Málek method}

The y $(\alpha)$ diagram for COC was plotted, and the kinetic model was used based on Table 2. To calculate the kinetic parameters by Málek method, after solving the equations, the kinetic parameters $(E),(\ln A),(n)$ were obtained to simulate the TG and DTG diagrams. Fig. 5 was the TG diagram obtained from the laboratory data and the diagram simulated by the Málek method at thermal rates of 5, 10, and $20{ }^{\circ} \mathrm{C} / \mathrm{min}$. (Fig. 5 and 6). 


\subsubsection{Kinetic parameters by fitting method}

. Using Fig. 7 and 8 the kinetic parameters for COC were calculated and presented in the table 5 by Malek and fitting methods. The nth-order reaction method in modeling the simulated graph for the TG and DTG diagrams for the COC did not work well. (Fig. 9)

\subsection{Calculation of activation energy for PMMA/COC40 alloy}

The activation energy (E) changed with conversion percentage (a). Therefore, we concluded that the degradation consisted of two stages, the first stage related to PMMA, which changed the activation energy in the range of $200-200 \mathrm{KJmol}^{-1}$ to $60 \%$ for conversion percentages, and the second stage related to COC, which changed approximately $70 \%$ after conversion percentage. , Activation energy increased to $250 \mathrm{KJmol}^{-1}$. The activation energy related to the COC degradation phase was also calculated by Kissinger method. TG and DTG diagrams obtained from laboratory data and simulated diagrams in PMMA/COC40 alloy at thermal rates of 5, 10, and 20 ${ }^{\circ} \mathrm{C}$ (Fig. 10 and 11), and kinetic parameters were shown in Table 6.

\subsection{Calculation of activation energy after irradiation for PMMA/COC40 alloy}

The activation energy (E) changed with conversion percentage (a). Therefore, degradation consisted of two stages. In the alloy, PMMA (as a non-gel polymer) in irradiation had a greater tendency to degrade than crosslinking, and on the other hand, COC showed a greater tendency to crosslink. Therefore, crosslinking of COC chains in the alloy affected the PMMA chains and increased the PMMA activation energy after irradiation to prevent the mass and heat generated by the COC crosslinked chains.

\subsubsection{Kinetic parameters after irradiation}

TG and DTG diagrams obtained from laboratory data and simulated diagrams in PMMA/COC40 alloy at thermal rates of 5,10, and $20^{\circ} \mathrm{C}$ after irradiation (Fig. 12 and 13), and kinetic parameters were shown in Table 7.

\section{Discussion}

\subsection{Mechanism of thermal degradation of PMMA}

In thermal degradation, polymethyl methacrylate, losing weight occurs in one, two, three, or four stages. The stages of thermal degradation of PMMA vary according to the molecular structure of the polymer and how the polymer is produced in the laboratory. Weak bonds in the polymer structure break at low temperatures, while stronger bonds have greater thermal stability. Degradation begins with weak bond rupture and accidental chain rupture. Weak bonds are formed during polymerization by the addition of an additional monomer head-to-head bond or by a disproportionate partition reaction during the termination step, which produces the end of the vinylidene chain [24].

The thermal degradation of PMMA, which is produced by free-radical polymerization, occurs in three stages. The first stage occurred in the temperature range of approximately $165-175^{\circ} \mathrm{C}$, in which the degradation is due to the head-to-head rupture of the weak $\mathrm{H}-\mathrm{H}$ bond. The second stage of degradation occurs at $225^{\circ} \mathrm{C}$ due to the rupture of unsaturated end groups that occur during the disproportionate partitioning reaction in the final stage. While other saturation ends groups maintain their thermal stability up to $300{ }^{\circ} \mathrm{C}$. The third stage of degradation occurs at temperatures above $300{ }^{\circ} \mathrm{C}$, due to accidental rupture of the polymer chain [25].

Hirata et al. [26] conducted a study on the thermal degradation of PMMA through free radical polymerization. During the research, slight weight loss occurred at temperatures below $200{ }^{\circ} \mathrm{C}$. Degradation at this stage was due to the escape of low molecular weight molecules such as additives or monomers left in the polymer chains. The second and most important stage of degradation at $290{ }^{\circ} \mathrm{C}$ due to the depropagation reaction was achieved through random chain rupture. In this investigation, thermal analysis was performed on $25-30 \mathrm{mg}$ of each sample. Substantial weight loss was observed between the temperature range of $150-190{ }^{\circ} \mathrm{C}$, which could be related to the volatility of low molecular weight molecules such as additives or monomers remaining in the polymer chain. The most important stage of degradation was weight loss at $280{ }^{\circ} \mathrm{C}$, which continued up to 480 ${ }^{\circ} \mathrm{C}$. At the end of the experiment, less than $0.5 \%$ of the remaining weight remained from PMMA degradation. This residual weight can be considered as ash. This ash resulted from the removal of the methoxycarbonyl side group, which produces an unsaturated conjugate system [27]. As mentioned, very slight weight loss occurred at $150{ }^{\circ} \mathrm{C}$, which can be ignored in modeling, so the data between the temperature ranges of $284-280{ }^{\circ} \mathrm{C}$ are used in modeling.

\subsection{Mechanism of thermal degradation pure COC}

Cyclic olefin copolymer is an engineered thermoplastic with some unique properties such as high TG, excellent transparency, and low moisture absorption. Studies on thermal stability and degradation kinetics have shown that COC, despite the low degradation starts temperature, narrower degradation temperature range, and also have a large amount of residual weight at the end of degradation, maintains the thermal stability of polyolefin 
materials well. This feature is related to the chemical structure and morphological properties of the COC as well as the effects of the branch. The onset temperature of COC degradation of HDPE is reported to be approximately between $430-410{ }^{\circ} \mathrm{C}$. In COC, chain separation occurred in a shorter time, it has a narrower degradation range than HDPE. Branch effects also cause changes in the reaction mechanism, so bonds adjacent to side groups have a higher rupture rate than PE bonds. Polyolefins are composed of the elements carbon and hydrogen, so very little of the remaining weight remains at the end of degradation. At the end of COC degradation, $2.5 \%$ by weight, black ash remains. This phenomenon is due to some network structures that are the reaction between radicals. The rate of radical formation is directly related to thermal stability, so the more thermally stable the radicals formed in the degradation process, the greater the number of lattice structures $[3,5]$. In the structure of Norbornene in the $\mathrm{COC}$, triple carbon hydrogens are separated more quickly and easily. Therefore, with increasing the percentage of triple carbon, the thermal stability decreases. As a result, at high Norbornene accumulations, the activation energy decreases due to the increase in the number of triple carbons. The thermal stability of COC under nitrogen is approximately $400{ }^{\circ} \mathrm{C}[28,29]$.

\section{Conclusion}

In this study, a study on thermal degradation kinetics of two types of thermoplastics, polymethyl methacrylate, cyclic olefin copolymers, and their alloys was performed before and after irradiation. As observed for the heat degradation reaction of polymethyl methacrylate and cyclic olefin copolymer, step-by-step kinetic calculations were performed starting with Kissinger method in which in both polymers, the degradation process was detected as a simple, single-step process and energy changes. Activation with conversion percentage was calculated for both polymers. It was observed that both PMMA and COC polymers are degraded in one step so that eventually COC leaves $2-5 \%$ by weight and PMMA less than $0.5 \%$ by weight. Based on the changes in the activation energy relative to the conversion percentage in the alloy, it was observed that the degradation is two-stage.

For the PMMA degradation process, a suitable kinetic model was proposed, in which the nth-order reaction method responded well to the other methods, and finally the kinetic parameters were calculated and the calorimetric curve of the proposed model was simulated. It was found that the experimental data showed a good agreement with the simulated curve. Similar calculations were performed for COC and the kinetic parameters were calculated using the Málek method at different heating rates and in the nitrogen medium. For PMMA / COC40 alloy, a suitable kinetic model was proposed before and after irradiation and finally, the kinetic parameters were calculated. In alloys, PMMA (as a non-gel polymer) tends to degrade more than crosslinked in irradiation, while COC tends to crosslink [6], so crosslinking COC chains in alloys on PMMA chains was effective and due to the mass inhibition of heat generated by COC crosslinked chains, the PMMA activation energy increased after irradiation. After irradiation due to crosslinking of COC chains, thermal stability decreased with increasing carbon triple percentage. As a result, at the high Norbornene accumulation due to the increase in the number of triple carbons, the activation energy decreased, so the calculated activation energy (Kissinger method) in the irradiated alloy was less than the alloy before irradiation.

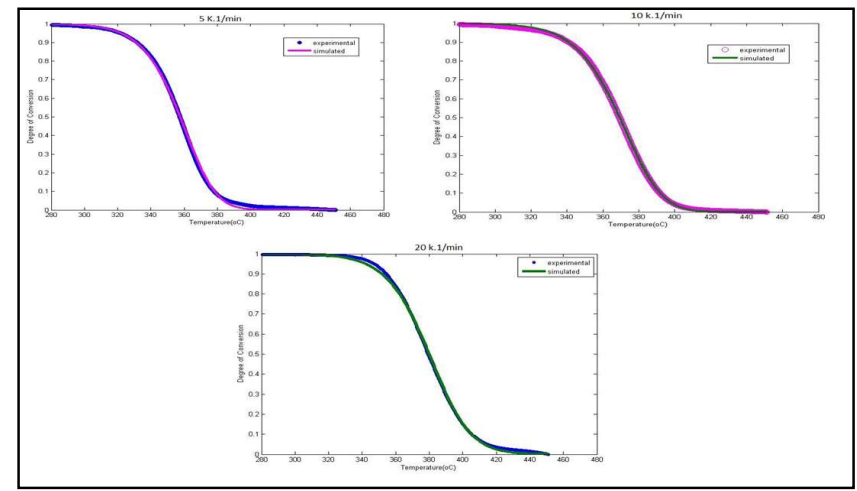

Fig. 1 TG diagrams obtained from laboratory data and nth-order reaction simulated diagrams at thermal rates 5,10,20 ${ }^{\circ} \mathrm{C} / \mathrm{min}$ (PMMA) 


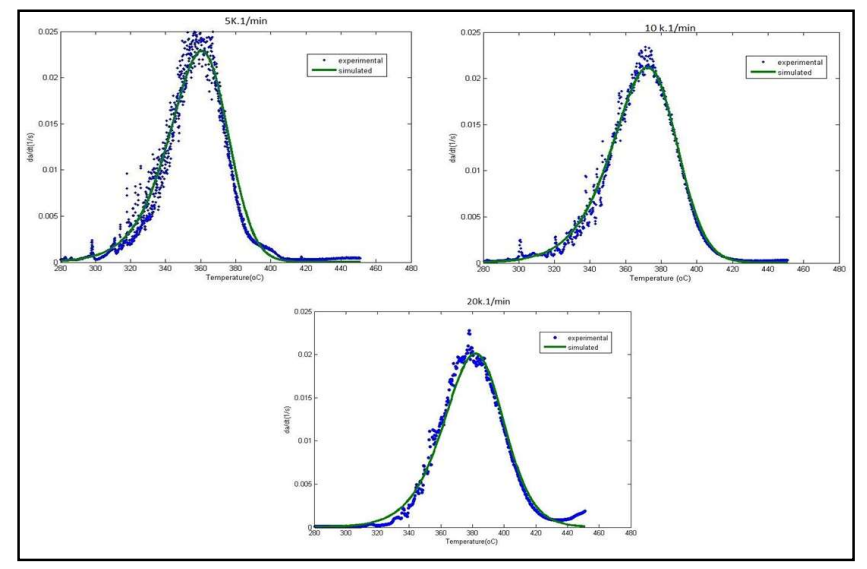

Fig. 2 DTG diagrams obtained from laboratory data and the nth-order reaction simulated diagrams at thermal rates of $5,10,20{ }^{\circ} \mathrm{C} / \mathrm{min}$ (PMMA)

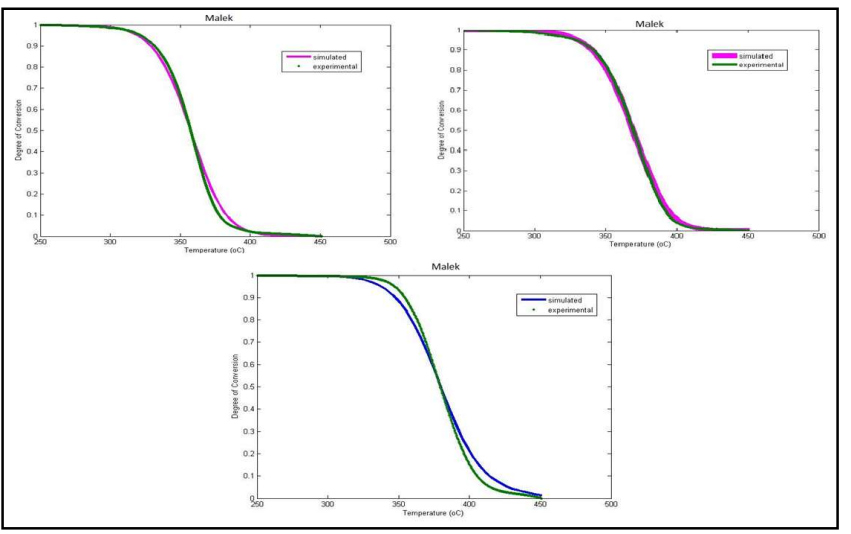

Fig. 3 TG diagrams obtained from laboratory data and diagrams simulated by the Málek method at thermal rates of 5,10,20 ${ }^{\circ} \mathrm{C} / \mathrm{min}(\mathrm{PMMA})$

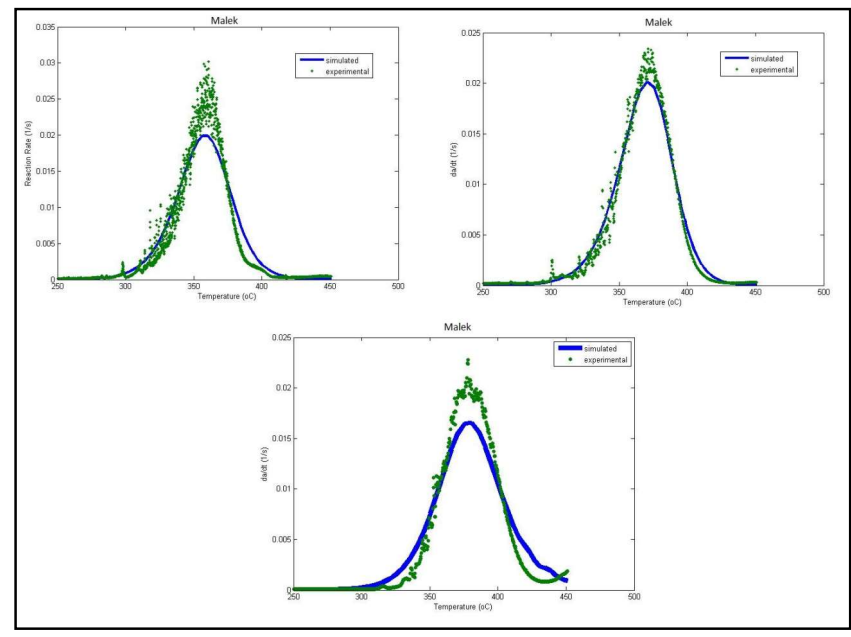

Fig. 4 DTG diagrams obtained from laboratory data and diagrams simulated by the Málek method at thermal rates of 5,10,20 ${ }^{\circ} \mathrm{C} / \mathrm{min}$ (PMMA) 


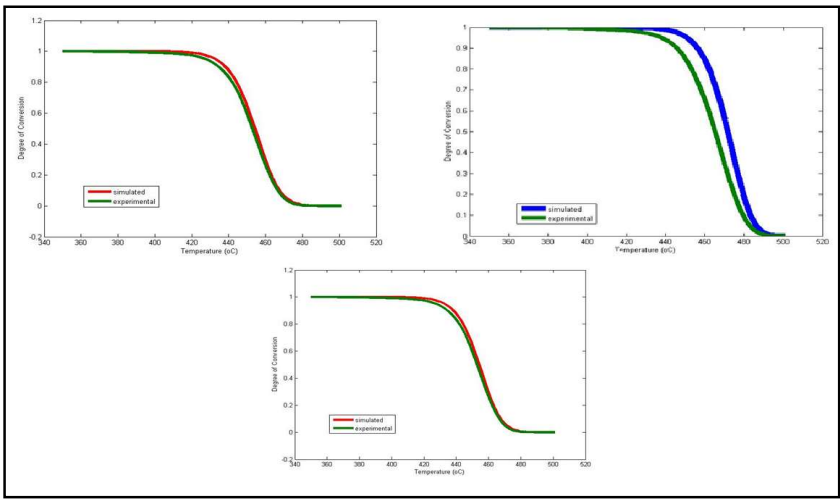

Fig. 5 TG diagrams obtained from laboratory data and diagrams simulated by the Málek method at thermal rates of 5,10,20 ${ }^{\circ} \mathrm{C} / \mathrm{min}(\mathrm{COC})$

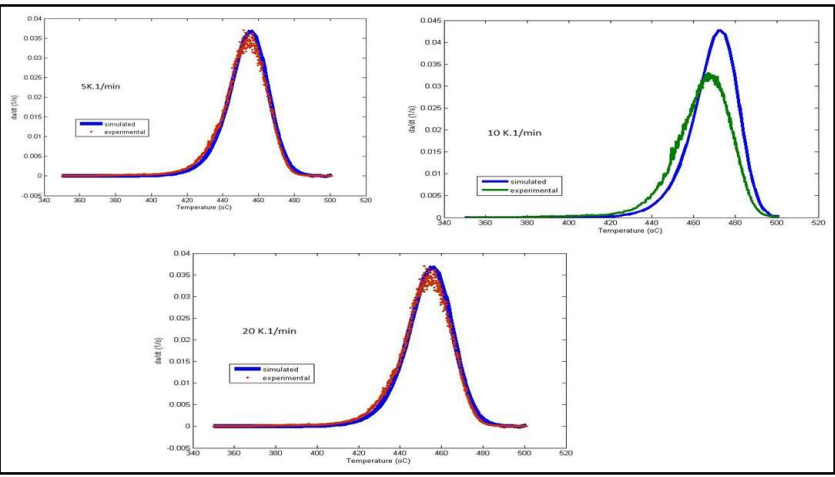

Fig. 6 DTG diagrams obtained from laboratory data and diagrams simulated by the Málek method at thermal rates of 5,10,20 ${ }^{\circ} \mathrm{C} / \mathrm{min}(\mathrm{COC})$

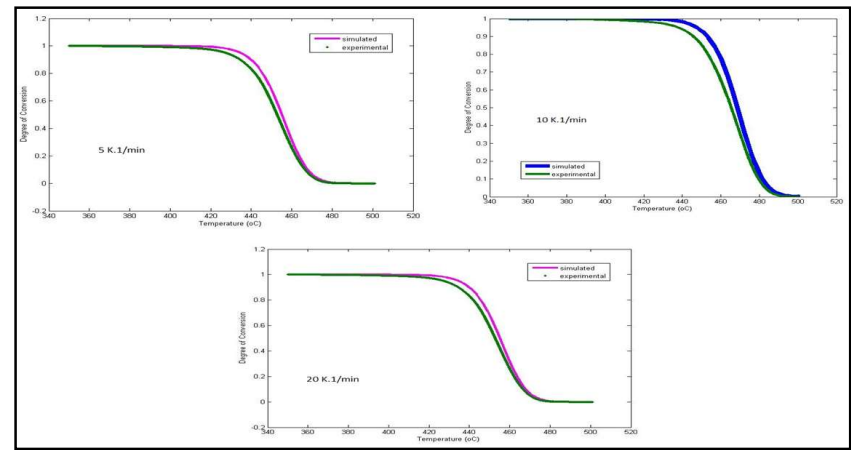

Fig. 7 TG diagrams obtained from laboratory data and diagrams simulated by the fitting method at thermal rates of 5,10,20 ${ }^{\circ} \mathrm{C} / \mathrm{min}(\mathrm{COC})$

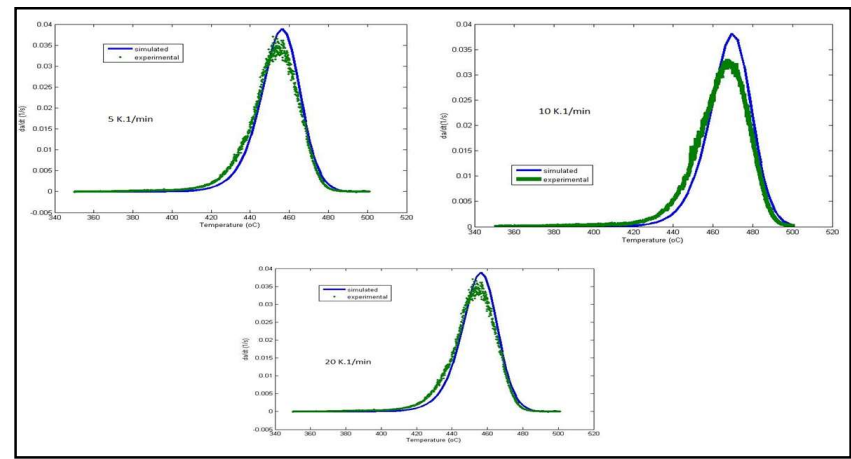

Fig. 8 DTG diagrams obtained from laboratory data and diagrams simulated by the fitting method at thermal rates of 5,10,20 ${ }^{\circ} \mathrm{C} / \mathrm{min}(\mathrm{COC})$ 


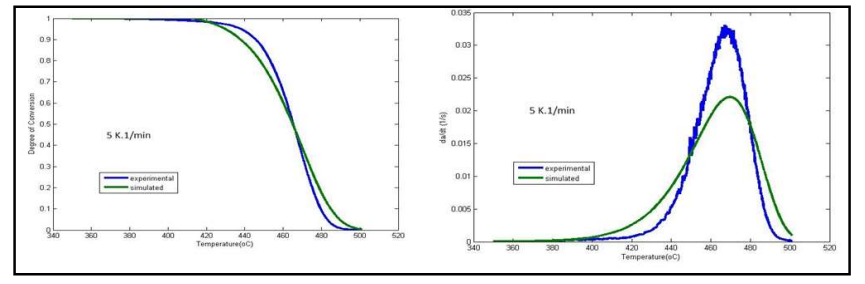

Fig. 9 DTG and TG diagrams from laboratory data and diagrams simulated by nth-order reaction method at $5{ }^{\circ} \mathrm{C}(\mathrm{COC})$

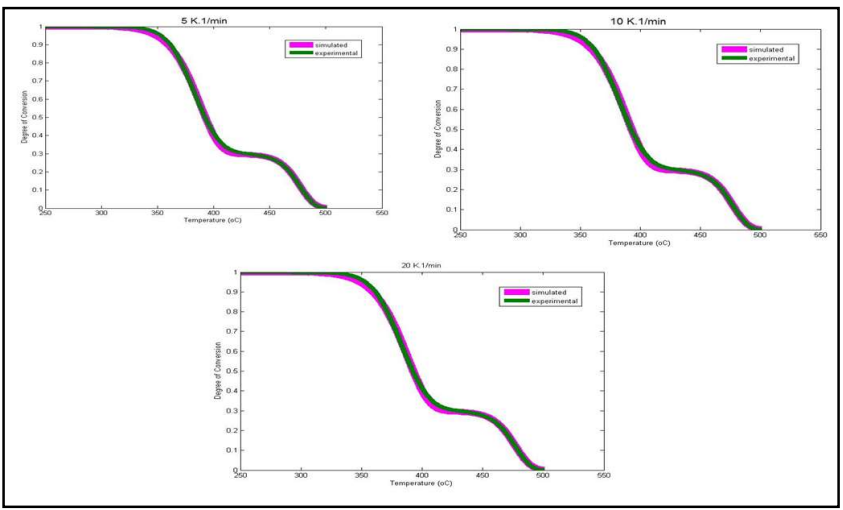

Fig. $10 \mathrm{TG}$ diagrams obtained from laboratory data and diagrams simulated at thermal rates of 5,10,20 ${ }^{\circ} \mathrm{C} / \mathrm{min}$ (PMMA/COC40)

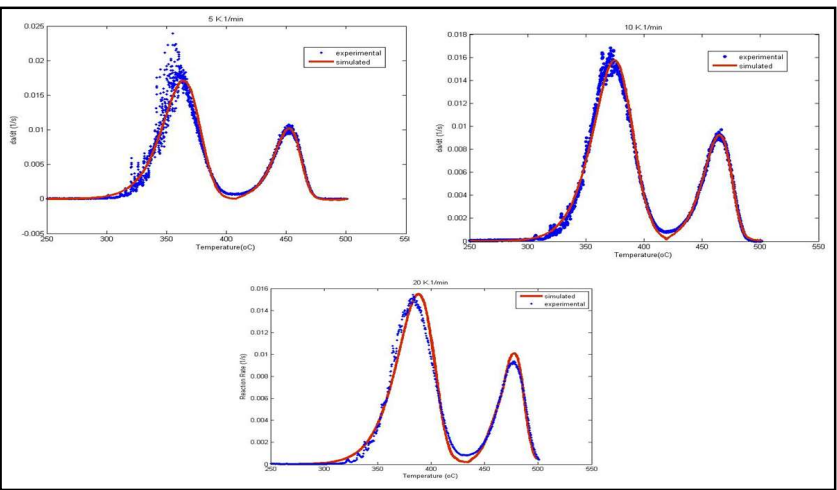

Fig. 11 DTG diagrams obtained from laboratory data and diagrams simulated at thermal rates of 5,10,20 ${ }^{\circ} \mathrm{C} / \mathrm{min}$ (PMMA/COC40)

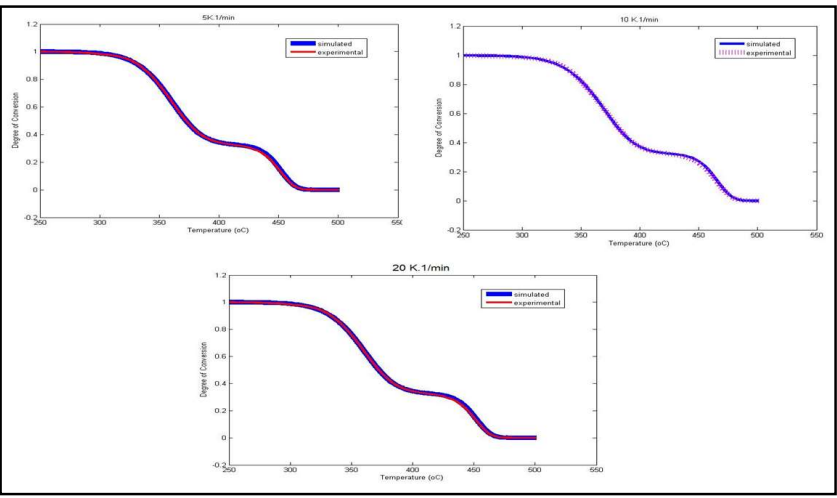

Fig. 12 TG diagrams obtained from laboratory data and diagrams simulated at thermal rates of $5,10,20{ }^{\circ} \mathrm{C} / \mathrm{min}$ after irradiation (PMMA/COC40) 


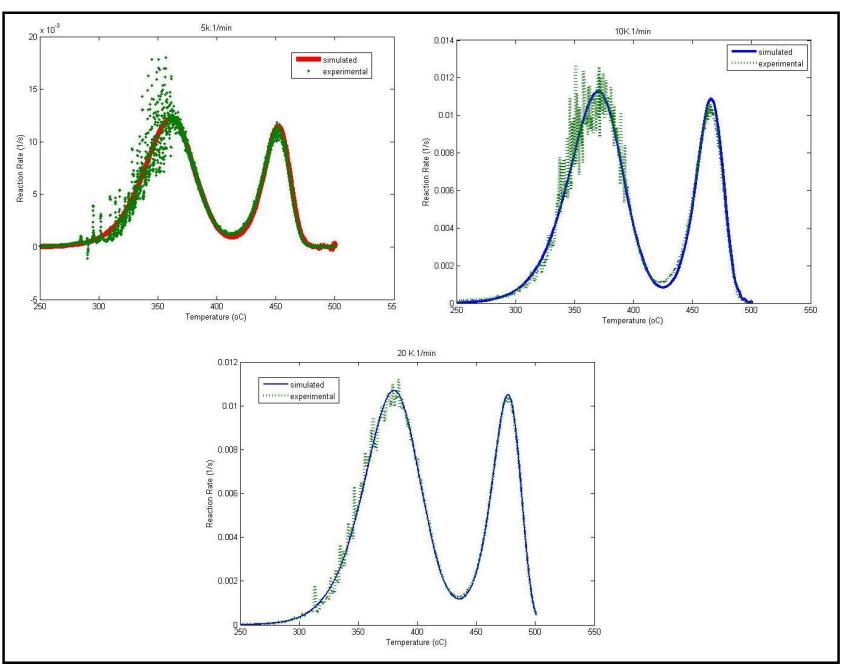

Fig. 13 DTG diagrams obtained from laboratory data and diagrams simulated at thermal rates of 5,10,20 ${ }^{\circ} \mathrm{C} / \mathrm{min}$ after irradiation (PMMA/COC40)

\begin{tabular}{clccc}
\multicolumn{1}{c}{ Table 1 Some physical physical properties of PMMA and COC by the manufacturer } \\
\hline \multicolumn{1}{c}{ Material } & \multicolumn{1}{c}{ Properties } & Unit & Value & Test standard \\
\hline \multirow{4}{*}{ PMMA } & Density & $\mathrm{g} / \mathrm{cm}^{3}$ & 1.19 & $\mathrm{D} 792$ \\
& Melt flow index & $\mathrm{g} / 10 \mathrm{~min}$ & 1.80 & $\mathrm{D} 1238$ \\
& Tensile strength at breaking point & $\mathrm{MP}$ & 7.03 & $\mathrm{D} 638$ \\
& Elongation at break point & $\%$ & 1.50 & $\mathrm{D} 638$ \\
& Izod hit (no notch) & $\mathrm{Kj} / \mathrm{m}^{2}$ & 12.00 & $\mathrm{D} 256$ \\
\hline \multirow{6}{*}{ COC } & Density & $\mathrm{g} / \mathrm{cm}^{3}$ & 1.02 & $\mathrm{D} 792$ \\
& Melt flow index & $\mathrm{g} / 10 \mathrm{~min}^{3}$ & 4.00 & $\mathrm{D} 1238$ \\
& Tensile strength at breaking point & $\mathrm{MP}$ & 60.00 & $\mathrm{D} 638$ \\
& Elongation at break point & $\%$ & 2.50 & $\mathrm{D} 638$ \\
& Izod hit (no notch) & $\mathrm{Kj} / \mathrm{m}^{2}$ & 15.00 & $\mathrm{D} 256$ \\
\hline
\end{tabular}

PMMA: polymethyl methacrylate COC: Cyclic Olefin Copolymer

Table 2 Commonly used kinetic models

\begin{tabular}{lcc}
\hline \multicolumn{1}{c}{ Models } & Symbols & $f(a)$ \\
\hline Johnson-Mehl-Avrami & JMA(n) & $n(1-\alpha)\left[-\left.\ln (1-\alpha)\right|^{1 / 1 / n}\right.$ \\
2D-reaction & R2 & $(1-\alpha)^{1 / 2}$ \\
3D-reaction & R3 & $(1-\alpha)^{1 / 3}$ \\
2D-diffusion & D2 & $1 /[-\ln (1-\alpha)]$ \\
Jander eqn. & D3 & $3 / 2(1-\alpha)^{2 / 3} /\left[1-(1-\alpha)^{2 / 3}\right]$ \\
Ginstling-Brounshtein & D4 & $3 / 2\left[(1-\alpha)^{-1 / 3}-1\right]$ \\
Reaction order & ROba) & $(1-\alpha)^{n}$ \\
Sesdk-Berggren & SB(m, n) & $\alpha^{m}(1-\alpha)^{n}$ \\
\hline
\end{tabular}

Table 3 PMMA peak points at the different thermal rates $5,10,20^{\circ} \mathrm{C} / \mathrm{min}$

\begin{tabular}{cc} 
Table 3 PMMA peak points at the different thermal rates $5,10,20^{\circ} \mathrm{C} / \mathrm{min}$ \\
\hline $\mathrm{B}\left({ }^{\circ} \mathrm{C}\right.$ min $\left.^{-1}\right)$ & T Peak $\left({ }^{\circ} \mathrm{C}\right)$ \\
\hline 5 & 358.483 \\
10 & 371.172 \\
20 & 378.447 \\
\hline
\end{tabular}


Table 4 Kinetic parameters calculated for pure PMMA

\begin{tabular}{ccccc}
\hline Method & Heating rate $\beta(\mathrm{K} / \mathrm{min})$ & $E_{a}(\mathrm{~kJ} / \mathrm{mol})$ & $\ln (A)$ & $n$ \\
\hline \multirow{3}{*}{ Malek } & 5 & 220.6616 & 36.7995 & 1.5788 \\
& 10 & 220.6616 & 36.6303 & 1.4541 \\
& 20 & 220.6616 & 36.8191 & 1.9547 \\
& Average & 220.6616 & 36.74963 & 1.662533 \\
\hline \multirow{3}{*}{ nth-reaction order } & 5 & 224.9941 & 37.5151 & 1.2683 \\
& 10 & 217.3064 & 35.9189 & 1.2885 \\
& 20 & 232.7724 & 38.9014 & 1.511 \\
& Average & 225.0243 & 37.44513 & 1.355933 \\
\hline
\end{tabular}

Table 5 Kinetic parameters calculated for COC

\begin{tabular}{cccccc}
\hline Method & Heating rate $\beta(\mathrm{K} / \mathrm{min})$ & $E_{a}(\mathrm{~kJ} / \mathrm{mol})$ & $\ln (A)$ & $n$ & $\mathrm{~m}$ \\
\hline \multirow{4}{*}{ Malek } & 5 & 241.69 & 31.614 & 1.084 & 0.5815 \\
& 10 & 241.69 & 31.4523 & 0.9487 & 0.4915 \\
& 20 & 241.69 & 31.5354 & 0.891 & 0.4374 \\
Fitting & Average & 241.69 & 31.5339 & 0.974567 & 0.503467 \\
& 5 & 241.69 & 31.6363 & 1.0798 & 0.607 \\
& 10 & 241.69 & 31.6300 & 1.0617 & 0.5929 \\
& 20 & 241.69 & 31.6511 & 0.9442 & 0.5289 \\
& Average & 241.69 & 31.63913 & 1.02857 & 0.576267 \\
\hline
\end{tabular}

Table 6 Kinetic parameters calculated for pure PMMA/COC40

\begin{tabular}{cccccc}
\hline Method & Heating rate $\beta(\mathrm{K} / \mathrm{min})$ & $E_{a}(\mathrm{~kJ} / \mathrm{mol})$ & $\ln (A)$ & $n$ & $\mathrm{~m}$ \\
\hline \multirow{3}{*}{ Malek ( COC) } & 5 & 258.8801 & 38.1468 & 0.9703 & 0.3985 \\
& 10 & 258.8801 & 38.0091 & 0.9646 & 0.3514 \\
& 20 & 258.8801 & 38.056 & 0.9891 & 0.3925 \\
\multirow{2}{*}{ nth-order reaction } & Average & 258.8801 & 38.07063 & 0.974667 & 0.3808 \\
\hline \multirow{2}{*}{ (PMMA) } & 5 & 226.2954 & 37.5151 & 1.1163 & $*$ \\
& 10 & 230.1399 & 38.2082 & 1.2788 & $*$ \\
& 20 & 207.337 & 33.7493 & 1.0051 & $*$ \\
& Average & 221.2574 & 36.49087 & 1.1334 & $*$ \\
\hline
\end{tabular}

Table 7 Kinetic parameters calculated for pure PMMA/COC40 after irradiation

\begin{tabular}{cccccc}
\hline Method & Heating rate $\beta(\mathrm{K} / \mathrm{min})$ & $E_{a}(\mathrm{~kJ} / \mathrm{mol})$ & $\ln (A)$ & $n$ & $\mathrm{~m}$ \\
\hline \multirow{2}{*}{ Malek } & 5 & 244.8298 & 35.9005 & 1.0037 & 0.4586 \\
& 10 & 244.8298 & 35.792 & 1.0098 & 0.4542 \\
& 20 & 244.8298 & 35.7848 & 0.955 & 0.4109 \\
& Average & 244.8298 & 35.82577 & 0.9895 & 0.441233 \\
\hline \multirow{2}{*}{ nth-reaction orde } & 5 & 184.6899 & 29.6196 & 1.3721 & $*$ \\
(PMMA) & 10 & 168.4027 & 26.6619 & 1.2975 & $*$ \\
& 20 & 173.2103 & 27.7466 & 1.3828 & $*$ \\
\hline
\end{tabular}




\section{References}

[1] Patterson, D., et al. "Biodegradable Plastics Based on Sweet Potato Starch/Polyethylene Blends." INTERNATIONAL SAMPE SYMPOSIUM AND EXHIBITION. SAMPE; 1999, (2003).

[2] Production of plastics worldwide from 1950 to 2019. https://www.statista.com/statistics/282732/global-production-of-plasticssince-1950/

[3] Sudhakar, M., et al. "Marine microbe-mediated biodegradation of low-and high-density polyethylenes." International Biodeterioration \& Biodegradation 61.3 (2008): 203-213. https://doi.org/10.1016/j.ibiod.2007.07.011

[4] Jagger, Robert G., and Atef Okdeh. "Thermoforming polymethyl methacrylate." The journal of prosthetic dentistry 74.5 (1995): 542-545. https://doi.org/10.1016/S0022-3913(05)80359-2

[5] Lamonte, Ronald R., and Donal McNally. "Cyclic olefin copolymers." Advanced materials \& processes 159.3 (2001): 33-36.

[6] Kazemi M. "Fabrication of polymethyl methacrylate and cycloolefin copolymer alloy and study of their properties before and after irradiation". Master Thesis. Shiraz Azad University. (2013).

[7] T. Hancock, Brit. Pat. 4,768 (1823); ibid, 7,344 (1837).

[8] Capitain, Charlotte, et al. "Differential scanning calorimetry for quantification of polymer biodegradability in compost." International Biodeterioration \& Biodegradation 149 (2020): 104914. https://doi.org/10.1016/j.ibiod.2020.104914

[9] Ramakers-van Dorp, Esther, et al. "On merging DMA and microindentation to determine local mechanical properties of polymers." Polymer Testing 68 (2018): 359-364. https://doi.org/10.1016/j.polymertesting.2018.04.020

[10] Ollivier-Lamarque, Lucas, et al. "Dielectric analysis of water uptake in polymer coating using spatially defined Fick's law and mixing rule." Progress in Organic Coatings 148 (2020): 105846. https://doi.org/10.1016/j.porgcoat.2020.105846

[11] Koyanagi, Jun, et al. "Molecular dynamics simulation for the quantitative prediction of experimental tensile strength of a polymer material." Composites Part C: Open Access 2 (2020): 100041. https://doi.org/10.1016/j.jcomc.2020.100041

[12] WANG, Qing-guo, Xiao-wen LI, and Rong-shi CHENG. "Determination of Specific Refractive Index Increment (dn/dc) $\mu$ at a Constant Chemical Potential, for Solutions of Polymer in Mixed Solvents by the GPC Method." Chemical Research in Chinese Universities 23.5 (2007): 602-606. https://doi.org/10.1016/S1005-9040(07)60131-7

[13] Philipp, Martine, et al. "Polymerization-induced shrinkage and dynamic thermal expansion behavior during network formation of polyurethanes." Thermochimica Acta 677 (2019): 144-150. https://doi.org/10.1016/j.tca.2019.01.012

[14] Zhang, Jianxiang, et al. "Morphology modulation of polymeric assemblies by guest drug molecules: TEM study and compatibility evaluation." Polymer 50.7 (2009): 1778-1789. https://doi.org/10.1016/j.polymer.2009.02.004

[15] Čalkovský, Martin, et al. "Comparison of segmentation algorithms for FIB-SEM tomography of porous polymers: Importance of image contrast for machine learning segmentation." Materials Characterization 171 (1920): 110806. https://doi.org/10.1016/j.matchar.2020.110806

[16] Grassie, Norman. "Chemistry of high polymer degradation processes." (1956). https://doi.org/10.1002/pol.1957.1202511008

[17] Nikolaidis, Alexandros K., and Dimitris S. Achilias. "Thermal degradation kinetics and viscoelastic behavior of poly (methyl methacrylate)/organomodified montmorillonite nanocomposites prepared via in situ bulk radical polymerization." Polymers 10.5 (2018): 491. https://doi.org/10.3390/polym10050491

[18] Azimi.H, Rezae.M,"kinetics study of Thermal degradation of Poly methyl Metacrylate and polystyrene", Iranian Chemical Engineering Journal-Vol, 8-No, 41(2009).

[19] Málek, Jiř́. "The kinetic analysis of non-isothermal data." Thermochimica acta 200 (1992): 257-269. https://doi.org/10.1016/0040-6031(92)85118-F

[20] Vyazovkin, Sergey, et al. "ICTAC Kinetics Committee recommendations for performing kinetic computations on thermal analysis data." Thermochimica acta 520.1-2 (2011): 1-19. https://doi.org/10.1016/j.tca.2011.03.034

[21] Senum, G.I., Yang, R.T. Rational approximations of the integral of the Arrhenius function. Journal of Thermal Analysis 11, 445447 (1977). https://doi.org/10.1007/BF01903696

[22] Judah S (2021). Runge Kutta 4th order ode (https://www.mathworks.com/matlabcentral/fileexchange/29851-runge-kutta-4thorder-ode), MATLAB Central File Exchange. Retrieved January 29, 2021.

[23] Murthy, Z. V. P. "Nonlinear Regression: Levenberg-Marquardt Method." Encyclopedia of Membranes, E. Dioli and L. Giorno, Eds. Berlin: Springer (2014): 1-3. https://doi.org/10.1007/978-3-642-40872-4 1656-1

[24] Ferriol, M., et al. "Thermal degradation of poly (methyl methacrylate)(PMMA): modelling of DTG and TG curves." Polymer degradation and stability 79.2 (2003): 271-281. https://doi.org/10.1016/S0141-3910(02)00291-4 
[25] Manring, Lewis E. "Thermal degradation of poly (methyl methacrylate). 4. Random side-group scission." Macromolecules 24.11 (1991): 3304-3309. https://doi.org/10.1021/ma00011a040

[26] Hirata, Toshimi, Takashi Kashiwagi, and James E. Brown. "Thermal and oxidative degradation of poly (methyl methacrylate): weight loss." Macromolecules 18.7 (1985): 1410-1418. https://doi.org/10.1021/ma00149a010

[27] Pielichowski, Krzysztof, and James Njuguna. Thermal degradation of polymeric materials. iSmithers Rapra Publishing, 2005.

[28] León-Molina, Helia Bibiana, Mario Gutierrez-Villarreal, and Ricardo Acosta. "Kinetic study of the Thermal and Thermooxidative degradation of Ethylene-Norbornene Copolymers." https://doi.org/10.1007/s11144-017-1256-1

[29] Helia Bibiana León-Molina, Mario Gutierrez-Villarreal, Ricardo Acosta Centro de Investigación en Química Avanzada CIQA, Boulevard Enrique Reyna Hermosillo N 140. C.P. 25253. Saltillo - Coahuila, México. 